\title{
Analysis of Erbium and Vanadium Diffusion in Porous Silicon Carbide
}

\author{
Marina G. Mynbaeva, ${ }^{1}$ Evgeny L. Pankratov, ${ }^{2}$ Evgeniy N. Mokhov, ${ }^{1}$ and Karim D. Mynbaev ${ }^{1}$ \\ ${ }^{1}$ Ioffe Physical-Technical Institute of the Russian Academy of Sciences, 26 Polytechnicheskaya Street St., Petersburg 194021, Russia \\ ${ }^{2}$ Mathematical Department, Faculty of Radiophysics, Nizhny Novgorod State University, 65 Il'insky Street, \\ Nizhny Novgorod 603950, Russia \\ Correspondence should be addressed to Karim D. Mynbaev, mynkad@mail.ioffe.ru
}

Received 5 March 2012; Revised 1 June 2012; Accepted 15 June 2012

Academic Editor: Jörg Fink

Copyright (๑) 2012 Marina G. Mynbaeva et al. This is an open access article distributed under the Creative Commons Attribution License, which permits unrestricted use, distribution, and reproduction in any medium, provided the original work is properly cited.

\begin{abstract}
Experimental data on diffusion of erbium and vanadium in porous and nonporous silicon carbide at 1700 and $2200^{\circ} \mathrm{C}$ have been used for modelling diffusion in porous $\mathrm{SiC}$. It is shown that the consideration of pore structure modification under annealing via vacancy redistribution allows for satisfactory description of dopant diffusion. As expected, important contribution to the diffusion in the porous medium is found to be made by the walls of the pores: in SiC, the vacancy surface diffusion coefficient on the walls appears to exceed that in the bulk of the material by an order of magnitude. When thermal treatment transforms pore channels into closed voids, pathways for accelerated diffusion cease to exist and diffusion rates in porous and nonporous SiC become similar.
\end{abstract}

\section{Introduction}

Silicon carbide $(\mathrm{SiC})$ is a semiconductor that possesses properties highly suitable for high-power, high-frequency, and high-temperature microelectronic applications. For a long time, selective doping in $\mathrm{SiC}$ technology has been implemented mostly with ion implantation. Diffusion doping was considered to be impractical due to low diffusion coefficients of impurities at temperatures below $1800^{\circ} \mathrm{C}$, and the interest in diffusion in $\mathrm{SiC}$ was generally related to applications requiring "drive-in" annealing after implantation (see, e.g., [1-3]). Papers devoted to "classic" thermal diffusion in SiC were mostly limited to early years $[4,5]$. However, recent achievements in fabrication of SiC-based microelectronic devices using diffusion doping $[1,2,6]$ stirred renewed interest in diffusion in SiC.

Diffusion in $\mathrm{SiC}$ is believed to proceed mostly via kickout or vacancy-related mechanisms [1-5]. Vacancies define many physical properties of $\mathrm{SiC}[7,8]$, and one of the current subjects of research is their contribution to the formation and properties of porous $\mathrm{SiC}$, a material with many prospective applications $[9,10]$. The very formation of porous structure in $\mathrm{SiC}$ is believed to proceed via vacancy migration and clustering [11], and there are all grounds to believe that experimentally observed modification of the structure under thermal annealing $[12,13]$ is also due to vacancy redistribution. In this respect, diffusion in porous $\mathrm{SiC}$ represents a subject of special interest, as pores serve as the sources of vacancies, which should contribute to diffusion. In this paper, we consider diffusion in $\mathrm{SiC}$ with porous structure formed by the vacancy mechanism, and model a dynamic process, where diffusion of dopants proceeds against the background of fluxes of vacancies responsible for pore ripening during high-temperature annealing.

\section{Experimental Details}

To form the porous structure, $6 \mathrm{H}-\mathrm{SiC}$ wafers were subjected to surface anodization in HF. The anodization mode was chosen such as to ensure formation of pores via vacancyrelated mechanism, with no chemical etching of the surface involved [11]. As a result of anodization, porous layers were formed with thickness significantly greater than the expected diffusion depth. The dopants were erbium and vanadium. Erbium doping of $\mathrm{SiC}$ is attractive for infrared applications relying on radiative intrashell transitions in Er ions 
placed in semiconductor lattice [14], while vanadium is a dopant of choice for fabricating semi-insulating SiC [15]. In each experiment, the dopant was simultaneously introduced into SiC layer with a "nanoporous" structure, and into a reference sample of nonporous SiC. The diffusion was carried out in the so-called sandwich-type cell. Conditions in the cell prevent the crystal surface from decomposition in the course of high-temperature annealing, thus making possible quasi-equilibrium diffusion doping of nonporous $\mathrm{SiC}$ at temperatures $T \leq 2600^{\circ} \mathrm{C}$ [5]. The diffusion was carried out at $T=1700$ and $2200^{\circ} \mathrm{C}$ for a period of time of 30 and $120 \mathrm{~min}$. The dopant profiles were investigated using secondary ion mass spectroscopy (SIMS).

\section{Experimental Results}

To follow changes in the porous structure induced by the high temperature, a control set of samples was first subjected to thermal anneal under the same conditions, which were later used for diffusion. Figure 1 shows a typical example of the modification of porous structure in $6 \mathrm{H}-\mathrm{SiC}$ as a result of anneal at $T=1700^{\circ} \mathrm{C}$ for 120 and $240 \mathrm{~min}$. As can be seen, initial porous structure, which appeared in a form of branched thin pores with the pore stem diameter of 30 to $40 \mathrm{~nm}$ (a), after $120 \mathrm{~min}$ of anneal evolved into a set of "cylinders" with the diameter of 100 to $200 \mathrm{~nm}$ (b). Further annealing transformed the "cylinders" into closed voids, with some voids showing tendency to faceting (c). The pattern of porous structure after anneal at $2200^{\circ} \mathrm{C}$ for $120 \mathrm{~min}$ was similar to that shown in Figure 1(c), as with temperature increasing the modification of porous structure proceeds faster [13]. According to Cheremskoy et al. [16], the closing and faceting of the voids confirm that pore modification proceeds according to ripening mechanism, and it is due to vacancy redistribution.

SIMS measurements with depth profiling, performed both after the above anneals and dopant diffusion, did not reveal any serious deviation from $\mathrm{SiC}$ stoichiometry in the processed samples. The examples of dopant diffusion profiles are presented in Figure 2. As was shown elsewhere [17], only a small part of such profiles related to the area close to the surface could be fitted with complementary error function, which testified to the concentration-dependent diffusion. Figure 2(a) presents erbium profiles in porous and nonporous $\mathrm{SiC}$ after diffusion at $2200^{\circ} \mathrm{C}$ for 30 and $120 \mathrm{~min}$. As is seen, the erbium surface concentration in the samples was close to $3 \times 10^{18} \mathrm{~cm}^{3}$. For samples diffused for $30 \mathrm{~min}$, we observed a difference in the diffusion depth in porous and nonporous specimens. For samples diffused for $120 \mathrm{~min}$, this difference became somewhat larger, but considering the difference in the surface concentration, not as large as could be expected if the "classic" inverse square root diffusion law was applicable.

A similar situation was observed with vanadium diffusion at $2200^{\circ} \mathrm{C}$. For all the diffused samples, the vanadium surface concentration was $\sim 2 \times 10^{17} \mathrm{~cm}^{-3}$ (Figure 2(b)). The short-time (30 $\mathrm{min}$ ) diffusion yielded diffusion depth in porous $\mathrm{SiC}$ larger than that in nonporous material. Increasing the anneal time to $120 \mathrm{~min}$, the difference in diffusion depth in porous and nonporous specimens remained almost the same.

\section{Modelling}

For modelling, let us consider diffusion of impurity atoms into a porous layer. The diffusion starts at the moment of time $t=0$ and proceeds through an interface with $x=0$. It can be described with the second Fick law written in the following form:

$$
\begin{aligned}
\frac{\partial C(x, y, z, t)}{\partial t}= & \operatorname{div}\left\{D_{C} \cdot \operatorname{grad}[C(x, y, z, t)]\right\} \\
& +\operatorname{div}\left\{\frac{D_{C S}}{\bar{V} k T} \cdot \operatorname{grad}[\mu(x, y, z, t)]\right\},
\end{aligned}
$$

with the following boundary and initial conditions:

$$
\begin{aligned}
& C(0, y, z, 0)=C_{0}, \quad C(x>0, y, z, 0)=0, \\
& \left.\frac{\partial C(x, y, z, t)}{\partial x}\right|_{x=L_{x}}=\left.\frac{\partial C(x, y, z, t)}{\partial y}\right|_{y=0} \\
& =\left.\frac{\partial C(x, y, z, t)}{\partial y}\right|_{y=L_{y}} \\
& =\left.\frac{\partial C(x, y, z, t)}{\partial z}\right|_{z=0} \\
& =\left.\frac{\partial C(x, y, z, t)}{\partial z}\right|_{z=L_{z}}=0, \\
& C(0, y, z, t)=C_{0}
\end{aligned}
$$

where $k=1.38 \cdot 10^{-23} \mathrm{JK}^{-1}$ is the Boltzmann constant, $\bar{V}$ is molar volume; $\mu(x, y, z, t)=R \cdot T \cdot \ln \left(V_{2} / V_{1}\right)$ is the chemical potential, $V_{1}$ and $V_{2}$ are the initial and final pore volumes, respectively, $R=8.31 \mathrm{~J} \mathrm{~mole}^{-1} \mathrm{~K}^{-1}$ is the molar gas constant, $L_{x}, L_{y}$, and $L_{z}$ are the dimensions of doped sample, $C(x, y, z, t)$ is the impurity concentration, and $D_{C}$ and $D_{C S}$ are the diffusion coefficients in the bulk and on the surface (pore walls) of the material, respectively (see, e.g., [18]). As follows from the experimental data, we should consider the dependence of $D_{C}$ and $D_{C S}$ on the vacancy and dopant concentration. These dependences can be described using the functions suggested by Gotra [19], Zorin et al. [20], and Ryssel and Ruge [21], respectively,

$$
\begin{aligned}
D_{C V}= & D_{C V L}(T)\left[1+\xi_{V} \frac{C^{\eta}(x, y, z, t)}{P^{\eta}(x, y, z, T)}\right] \\
& \times\left[1+\varsigma_{V} \frac{V(x, y, z, t)}{V_{\infty}}\right],
\end{aligned}
$$




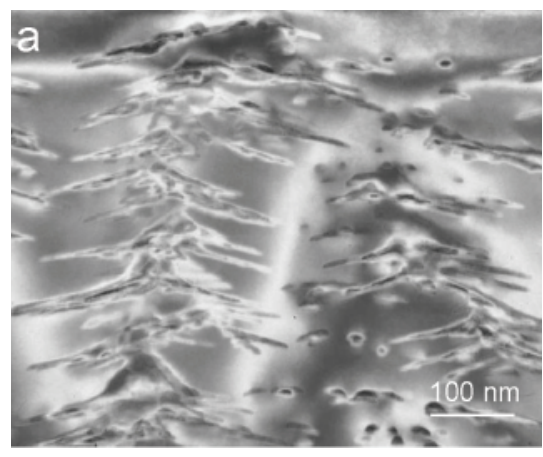

(a)

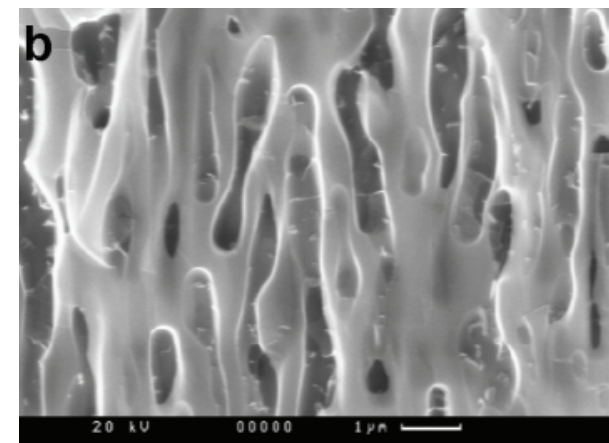

(b)

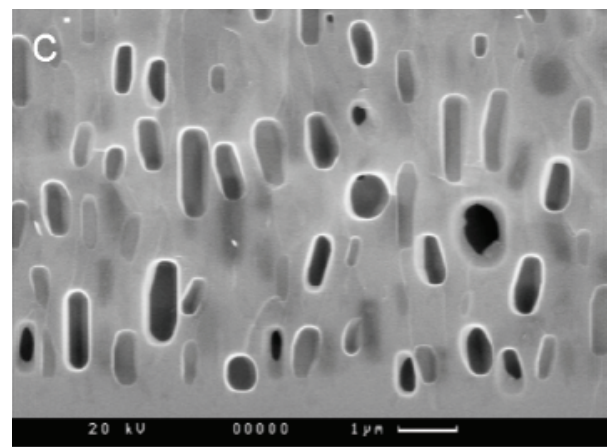

(c)

FIGURE 1: Transmission (a) and scanning ((b) and (c)) electron microscopy images of the cleft edge of porous layer in SiC: straight after anodization (a), after $1700^{\circ} \mathrm{C}$ anneal for $120 \mathrm{~min}(\mathrm{~b})$, and after $1700^{\circ} \mathrm{C}$ anneal for $240 \mathrm{~min}$ (c).

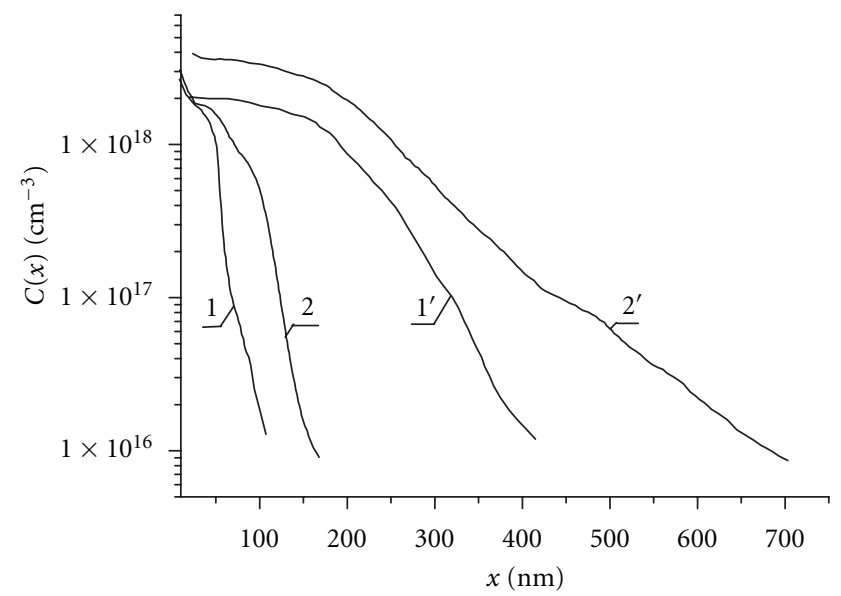

(a)

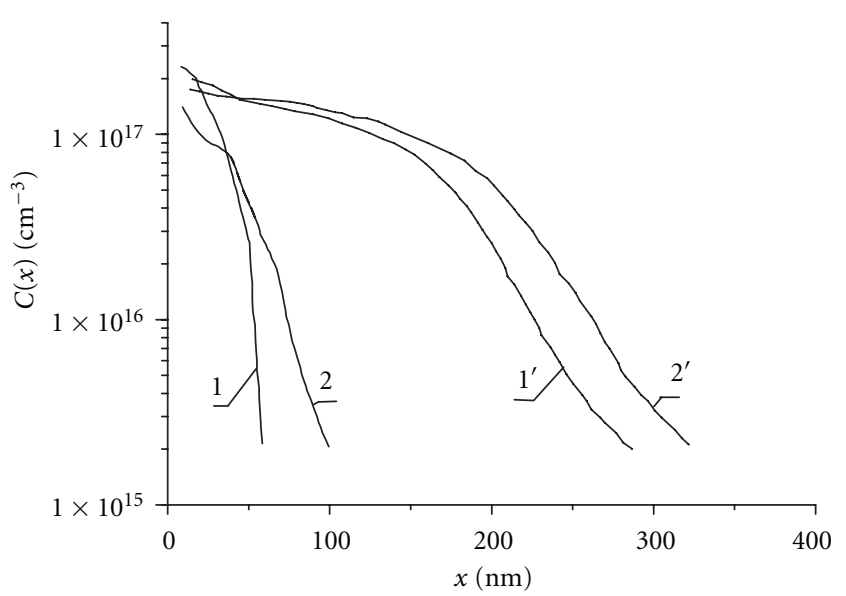

(b)

FIGURE 2: Experimental profiles showing erbium (a) and vanadium (b) concentration versus depth after diffusion at $2200^{\circ} \mathrm{C}$ for 30 min (curves 1 and 2) and $120 \mathrm{~min}$ (curves $1^{\prime}$ and $2^{\prime}$ ), in: 1: nonporous $\mathrm{SiC}, 2$ : porous $\mathrm{SiC}$.

$$
\begin{aligned}
D_{C S}= & D_{C S L}(T)\left[1+\xi_{S} \frac{C^{\eta}(x, y, z, t)}{P^{\eta}(x, y, z, T)}\right] \\
& \times\left[1+\varsigma_{S} \frac{V(x, y, z, t)}{V_{\infty}}\right] .
\end{aligned}
$$

Here, $D_{C L}(T)$ and $D_{C S L}(T)$ (where index Vrelates to diffusion in bulk and $S$ relates to surface diffusion) are linear (i.e., independent on dopant concentration, the linearity being indicated by index $L$ ) coefficients of the approximations. These (as well as solubility limits) have been extracted from the experimental data presented earlier [17]. The second multipliers in (3) describe concentration dependence of diffusion coefficients [19]. $P(x, y, z, T)$ is the dopant solubility limit. Value of the parameter $\eta$ (which is equal to an integer value in the range of 1 to 3 [19]) depends on the interaction between dopant atoms and defects $[19,22]$. The 
third multipliers in (3) describe vacancy dependences of the diffusion coefficients $[20,21] . V_{\infty}$ is the equilibrium vacancy distribution. Four fitting parameters, $\xi_{V}, \xi_{S}, \zeta_{V}, \zeta_{S}$ can be considered as scale multipliers of the limit of solubility of dopant and equilibrium vacancy concentration. The vacancy concentration can be described as follows:

$$
\begin{aligned}
\frac{\partial V(x, y, z, t)}{\partial t}= & \operatorname{div}\left\{D_{V}(T) \cdot \operatorname{grad}[V(x, y, z, t)]\right\} \\
& +\operatorname{div}\left\{\frac{D_{V S}(T)}{\bar{V} k T} \cdot \operatorname{grad}[\mu(x, y, z, t)]\right\}
\end{aligned}
$$

with the initial and boundary conditions:

$$
\begin{aligned}
\left.V(x, y, z, t)\right|_{S_{1}+\vec{V}_{n} t} & =V_{\infty}\left(1+\frac{2 \gamma \omega}{k T \sqrt{x_{1}^{2}+y_{1}^{2}+z_{1}^{2}}}\right), \\
\left.V(x, y, z, t)\right|_{S_{2}} & =V_{\infty}, \\
V(x, y, z, 0) & =f_{V}(x, y, z) \\
= & V_{\infty}\left(\frac{2 \gamma \omega}{k T \sqrt{x_{1}^{2}+y_{1}^{2}+z_{1}^{2}}}\right. \\
& \left.\times e^{-x / x_{1}} e^{-y / y_{1}} e^{-z / z_{1}}-1\right),
\end{aligned}
$$

where $S_{1}$ designates initial pore surface, $S_{2}$ stands for the surface of the outward boundary of the porous area, $V_{n}$ is the absolute value of the velocity of the movement of the surface of the growing or shrinking pore [18], $D_{V}$ and $D_{V S}$ are volumetric and surface diffusion coefficients of vacancies; $\gamma$ is the specific surface energy [16], and $\omega=a^{3}$, where $a$ is interatomic distance. The appropriateness of using (4) for describing the evolution of a pore under annealing was considered in detail by Kitayama et al. [18]. The initial and boundary conditions for this equation represent threedimensional generalization of those given by Cheremskoy et al. [16], with the consideration of pore size changes.

A typical vacancy distribution in the vicinity of a pore, which was obtained as a result of modelling using (4) is shown in Figure 3. Here, " $x$ " is the coordinate across the pore: the maximum value of vacancy concentration corresponds to the center of the pore. The initial pore was regarded as a cylinder with the radius of $40 \mathrm{~nm}$. The parameters of modelling were chosen so that the simulated annealing would lead to the growth of pore "cylinder" with the diameter of $\sim 200 \mathrm{~nm}$, which corresponds to the experimental data presented in Figure 1(b). The figure shows how annealing leads to the increase in pore diameter, and confirms qualitative adequacy of our model for consideration of pore modification under heat treatment on the microscopic scale.

To consider the diffusion, let us transform the diffusion coefficients into the following form: $D_{L}(x, y, z, T)=D_{0 L}[1+$ $\left.\varepsilon_{L} g_{L}(x, y, z, T)\right]$ and $D_{V}(x, y, z, T)=D_{0 V}\left[1+\varepsilon_{V} g_{V}(T)\right]$, where $D_{0 L}$ and $D_{0 V}$ are average values of diffusion coefficients, $0 \leq \varepsilon_{L}<1,0 \leq \varepsilon_{V}<1,\left|g_{L}(x, y, z, T)\right| \leq 1$,

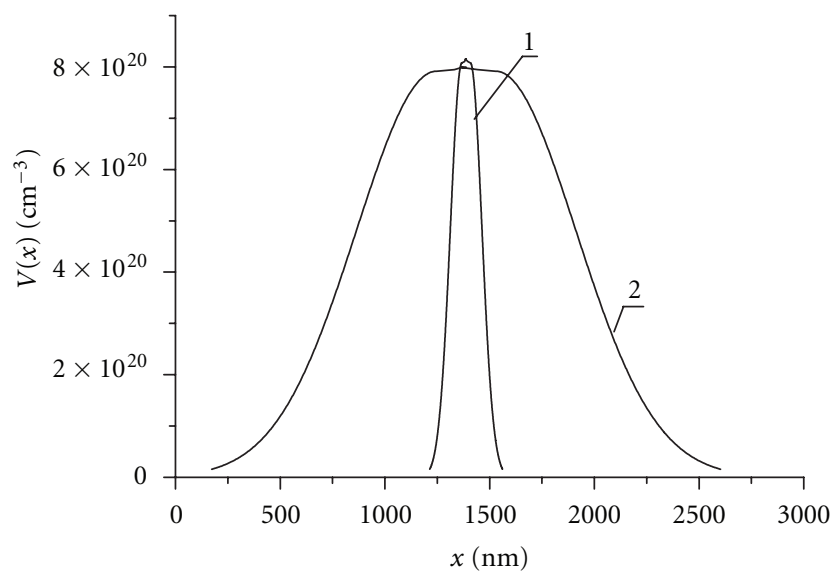

Figure 3: Modelled vacancy distribution in the vicinity of a pore in porous $\mathrm{SiC}$ before (curve 1) and after (curve 2) anneal at $1700^{\circ} \mathrm{C}$ for $120 \mathrm{~min}$.

$\left|g_{V}(T)\right| \leq 1$, (see, e.g., [22-24]). We determined solutions of (1) and (4) as the following power series:

$$
\begin{gathered}
C(x, y, z, t)=\sum_{i=0}^{\infty} \varepsilon_{L}^{i} \sum_{j=0}^{\infty} \xi^{j} C_{i j}(x, y, z, t), \\
V(x, y, z, t)=\sum_{i=0}^{\infty} \varepsilon_{V}^{i} V_{i}(x, y, z, t) .
\end{gathered}
$$

Substitution of the series (6) into (1), (4), and appropriate boundary and initial conditions gives us possibility to obtain equations for zero-order approximation of dopant and vacancy concentration $C_{00}(x, y, z, t)$ and $V_{0}(x, y, z, t)$, their corrections $C_{i j}(x, y, z, t)$ and $V_{i}(x, y, z, t)(i \geq 1, j \geq 1)$, and the boundary and initial conditions for all functions $C_{i j}(x, y, z, t)$ and $V_{i}(x, y, z, t)(i \geq 0, j \geq 0)$ :

$$
\begin{aligned}
& \frac{\partial C_{00}(x, y, z, t)}{\partial t} \\
& =D_{0 C} \Delta\left[C_{00}(x, y, z, t)\right] \\
& \quad+\operatorname{div}\left\{\frac{D_{C S}}{\bar{V} k T} \cdot \operatorname{grad}[\mu(x, y, z, t)]\right\}, \\
& \frac{\partial C_{i 0}(x, y, z, t)}{\partial t} \\
& \quad D_{0 C} \Delta\left[C_{i 0}(x, y, z, t)\right] \\
& \quad+D_{0 C} \operatorname{div}\left\{g_{L}(x, y, z, T)\right. \\
& \left.\quad \times \operatorname{grad}\left[C_{00}(x, y, z, t)\right]\right\}, \quad i \geq 1,
\end{aligned}
$$




$$
\begin{aligned}
& \frac{\partial C_{01}(x, y, z, t)}{\partial t} \\
& \quad=D_{0 C} \Delta\left[C_{01}(x, y, z, t)\right] \\
& \quad+D_{0 C} \operatorname{div}\left\{\frac{C_{00}^{\gamma}(x, y, z, t)}{P^{\gamma}(x, y, z, T)} \operatorname{grad}\left[C_{00}(x, y, z, t)\right]\right\},
\end{aligned}
$$

$$
\begin{array}{r}
\frac{\partial C_{02}(x, y, z, t)}{\partial t} \\
=D_{0 C} \operatorname{div}\left\{C_{01}(x, y, z, t) \frac{C_{00}^{\gamma-1}(x, y, z, t)}{P^{\gamma}(x, y, z, T)}\right. \\
\left.\quad \times \operatorname{grad}\left[C_{00}(x, y, z, t)\right]\right\} \\
+D_{0 C} \Delta\left[C_{02}(x, y, z, t)\right] \\
+D_{0 C} \operatorname{div}\left\{\frac{C_{00}^{\gamma}(x, y, z, t)}{P^{\gamma}(x, y, z, T)}\right. \\
\left.\quad \times \operatorname{grad}\left[C_{01}(x, y, z, t)\right]\right\},
\end{array}
$$

$\frac{\partial C_{11}(x, y, z, t)}{\partial t}$

$$
=D_{0 C} \Delta\left[C_{11}(x, y, z, t)\right]
$$$$
+D_{0 C} \operatorname{div}\left\{g_{L}(x, y, z, T)\right.
$$$$
\left.\times \operatorname{grad}\left[C_{01}(x, y, z, t)\right]\right\}
$$$$
+D_{0 C} \operatorname{div}\left\{\frac{C_{00}^{\gamma}(x, y, z, t)}{P^{\gamma}(x, y, z, T)}\right.
$$

$$
\left.\times \operatorname{grad}\left[C_{10}(x, y, z, t)\right]\right\},
$$

$$
\begin{aligned}
& \frac{\partial V_{0}(x, y, z, t)}{\partial t} \\
& =D_{0 V} \Delta\left[V_{0}(x, y, z, t)\right] \\
& \quad+\operatorname{div}\left\{\frac{D_{V S}}{\bar{V} k T} \cdot \operatorname{grad}[\mu(x, y, z, t)]\right\},
\end{aligned}
$$

$\frac{\partial V_{i}(x, y, z, t)}{\partial t}$

$$
\begin{aligned}
=D_{0 V} \Delta\left[V_{i 0}(x, y, z, t)\right] & \\
+D_{0 V} \operatorname{div}\left\{g_{V}(x, y, z, T)\right. & \\
& \left.\times \operatorname{grad}\left[V_{i-1}(x, y, z, t)\right]\right\}, \quad i \geq 1 \\
\left.\frac{\partial C_{i j}(x, y, z, t)}{\partial x}\right|_{x=0} & =\left.\frac{\partial C_{i j}(x, y, z, t)}{\partial x}\right|_{x=L_{x}} \\
& =\left.\frac{\partial C_{i j}(x, y, z, t)}{\partial y}\right|_{y=0}
\end{aligned}
$$

$$
\begin{aligned}
& =\left.\frac{\partial C_{i j}(x, y, z, t)}{\partial y}\right|_{y=L_{y}} \\
& =\left.\frac{\partial C_{i j}(x, y, z, t)}{\partial z}\right|_{z=0} \\
& =\left.\frac{\partial C_{i j}(x, y, z, t)}{\partial z}\right|_{z=L_{z}}=0,
\end{aligned}
$$

$$
\begin{aligned}
& C_{00}(0, y, z, 0)=C_{0}, \quad C_{i j}(0, y, z, 0)=0 \\
& (i \geq 1, j \geq 1) \\
& C_{i j}(x>0, y, z, 0)=0 \quad(i \geq 0, j \geq 0), \\
& C_{00}(0, y, z, t)=C_{0}, \quad C_{i j}(0, y, z, t)=0 \\
& (i \geq 1, j \geq 1) \text {, } \\
& \left.V_{0}(x, y, z, t)\right|_{S_{2}}=V_{\infty},\left.\quad V_{i}(x, y, z, t)\right|_{S_{2}}=0 \quad(i \geq 0) \text {, } \\
& \left.V_{00}(x, y, z, t)\right|_{S_{1}+\vec{V}_{n} t} \\
& =V_{\infty}\left(1+\frac{2 \gamma \omega}{k T \sqrt{x_{1}^{2}+y_{1}^{2}+z_{1}^{2}}}\right), \\
& \left.\frac{\partial V_{i}(x, y, z, t)}{\partial x}\right|_{x=0}=\left.\frac{\partial V_{i}(x, y, z, t)}{\partial x}\right|_{x=L_{x}} \\
& =\left.\frac{\partial V_{i}(x, y, z, t)}{\partial y}\right|_{y=0}=\left.\frac{\partial V_{i}(x, y, z, t)}{\partial y}\right|_{y=L_{y}} \\
& =\left.\frac{\partial V_{i}(x, y, z, t)}{\partial z}\right|_{z=0} \\
& =\left.\frac{\partial V_{i}(x, y, z, t)}{\partial z}\right|_{z=L_{z}}=0, \\
& \left.V_{i j}(x, y, z, t)\right|_{S_{1}+\vec{V}_{n} t}=0 \quad(i \geq 0, j \geq 0), \\
& V_{i j}(x, y, z, 0)=0 \quad(i \geq 0, j \geq 0), \\
& V_{00}(x, y, z, 0) \\
& =V_{\infty}\left(\frac{2 \gamma \omega}{k T \sqrt{x_{1}^{2}+y_{1}^{2}+z_{1}^{2}}}\right. \\
& \left.\times e^{-x / x_{1}} e^{-y / y_{1}} e^{-z / z_{1}}-1\right) \text {. }
\end{aligned}
$$

Solutions of (7) with conditions (8) are presented in the appendix. Though the values of the vacancy and dopant concentration can be determined with more accuracy, secondorder approximation usually gives satisfactory qualitative and some quantitative results (see, e.g., [22-24]). 


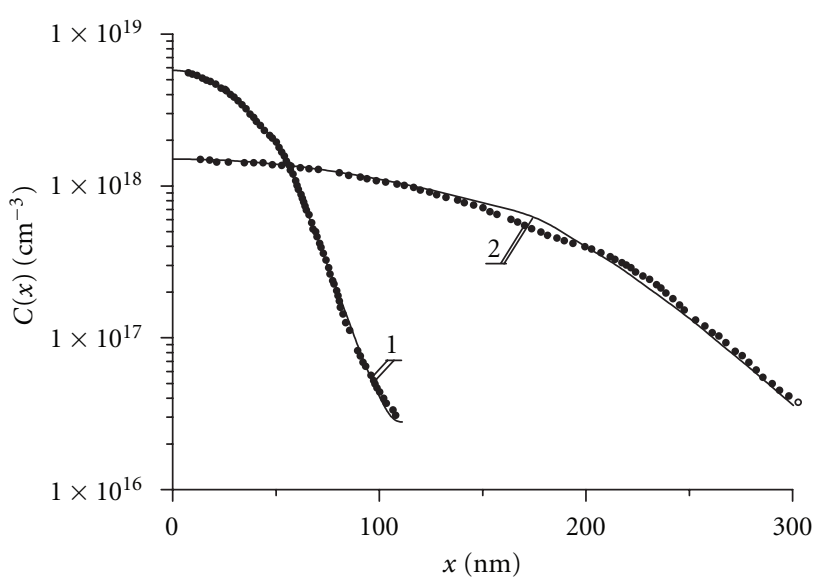

(a)

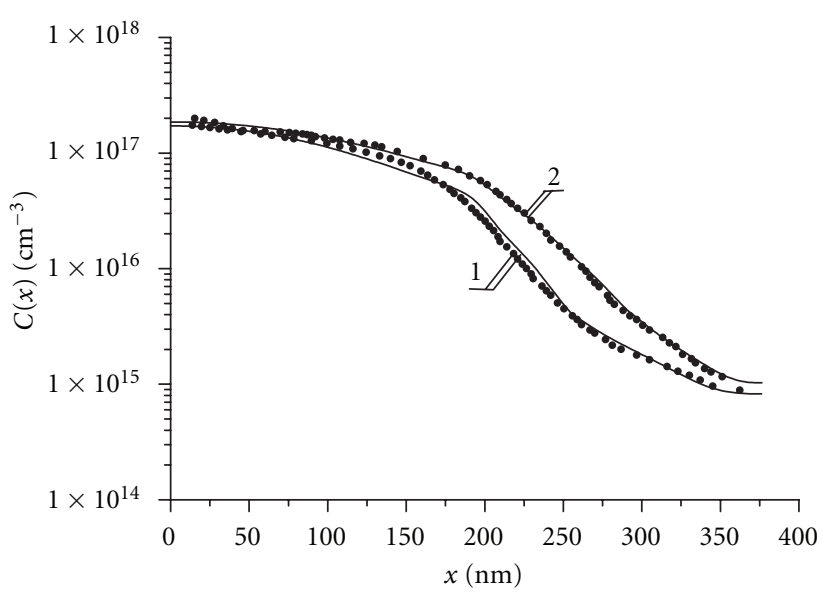

(b)

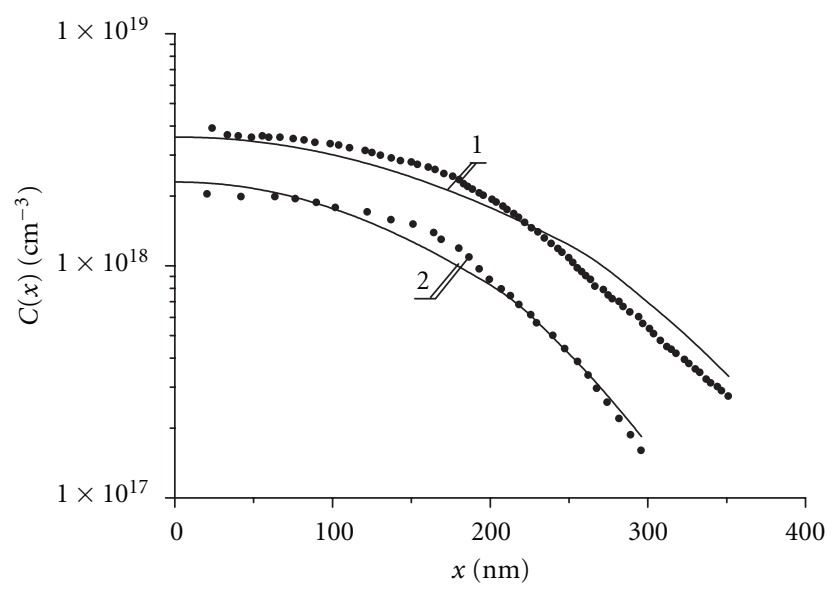

(c)

FIGURE 4: Distribution in non-porous $\mathrm{SiC}$ (curves 1) and porous $\mathrm{SiC}$ (curves 2) of: erbium after diffusion at $1700^{\circ} \mathrm{C}$ for $120 \mathrm{~min}$ (a), vanadium after diffusion at $2200^{\circ} \mathrm{C}$ for $120 \mathrm{~min}$ (b), and erbium after diffusion at $2200^{\circ} \mathrm{C}$ for $120 \mathrm{~min}$ (c). Points show experimental data, solid curves show results of fitting.

TABLE 1: Diffusion parameters obtained from the fitting.

\begin{tabular}{lcccc}
\hline Dopant & $D_{V}, \mathrm{~cm}^{2} \mathrm{~s}^{-1}$ & $D_{V S}, \mathrm{~cm}^{2} \mathrm{~s}^{-1}$ & $D_{C V L}, \mathrm{~cm}^{2} \mathrm{~s}^{-1}$ & $D_{C S L}, \mathrm{~cm}^{2} \mathrm{~s}^{-1}$ \\
\hline$V, 2200^{\circ} \mathrm{C}$ & $9.27 \cdot 10^{-14}$ & $2.78 \cdot 10^{-12}$ & $1.47 \cdot 10^{-14}$ & $2.36 \cdot 10^{-14}$ \\
Er, $1700^{\circ} \mathrm{C}$ & $2.48 \cdot 10^{-14}$ & $1.41 \cdot 10^{-13}$ & $3.58 \cdot 10^{-14}$ & $2.57 \cdot 10^{-14}$ \\
Er, $2200^{\circ} \mathrm{C}$ & $7.75 \cdot 10^{-14}$ & $8.65 \cdot 10^{-13}$ & $2.07 \cdot 10^{-13}$ & $8.42 \cdot 10^{-14}$ \\
\hline
\end{tabular}

\section{Discussion}

Let us analyze the experimental diffusion profiles using the relations obtained in the previous section. We will restrict our consideration to one dimension only, which is inward the porous layer (direction $x$ ). Considering the experimental results, we may assume that the results of 30 min-long anneals were somehow affected by transient processes, yet 120 min-long diffusion proceeded under "steady-state" conditions. This assumption is supported by the fact that the propagation of the diffusion fronts as presented in Figure 2 did not obey the inverse square root law. We fitted experimental profiles shown in Figure 2 by minimization of mean-squared error between experimental and theoretical results. The solid lines correspond to erbium profiles in porous and nonporous $\mathrm{SiC}$ after diffusion at $1700^{\circ} \mathrm{C}$ according to the procedure developed above for $120 \mathrm{~min}$, that is, long diffusion anneals. The parameters obtained are listed in Table 1 and the fitting curves are shown in Figure 4. Values of fitting parameters are presented in the Table 2. The parameters $\xi_{V}, \xi_{S}, \zeta_{V}$, and $\zeta_{S}$ give us corrections to experimental values of dopant solubility limit and equilibrium vacancy distribution. Their usefulness is determined by the fact that one can obtain quite a large difference between experimental data taken from different references. 
TABLE 2: Fitting parameters for Figure 4.

\begin{tabular}{|c|c|c|c|c|}
\hline Dopant & $\xi_{V}$ & $\xi_{S}$ & $\xi_{V}$ & $\xi_{S}$ \\
\hline Vanadium (in non-porous $\mathrm{SiC}$ ) & 0.32 & 0.23 & 0.21 & 0.33 \\
\hline Vanadium (in porous $\mathrm{SiC}$ ) & 0.29 & 0.24 & 0.23 & 0.21 \\
\hline Erbium (in non-porous $\mathrm{SiC}$ ) & 0.31 & 0.24 & 0.10 & 0.27 \\
\hline Erbium (in porous $\mathrm{SiC}$ ) & 0.33 & 0.30 & 0.22 & 0.19 \\
\hline
\end{tabular}

It follows from the results obtained that the vacancy diffusion coefficient on the surface of the pore walls $D_{V S}$ exceeds that in the bulk of the material $D_{V}$ approximately by an order of magnitude. Erbium appears to diffuse in $\mathrm{SiC}$ much faster than vanadium, seemingly due to stronger concentration dependence of its diffusion coefficient, that is, concentration dependence of diffusion coefficient leads to higher diffusion coefficient for erbium in comparison with that of vanadium. Summarizing the whole set of diffusion data obtained (including our earlier data [17]), it appears that when a short-term diffusion anneal is used, diffusion in porous $\mathrm{SiC}$ proceeds faster than that in nonporous $\mathrm{SiC}$ simply because porous structure does not have enough time to evolve into closed "cylinders." For long-term anneals, pathways for accelerated diffusion (pore walls) disappear as pore channels get transformed into closed voids, and diffusion coefficients in porous and nonporous $\mathrm{SiC}$ become close. This effect becomes more pronounced with increasing diffusion temperature. At low temperatures $\left(T<1000^{\circ} \mathrm{C}\right)$, the diffusion in porous $\mathrm{SiC}$ always proceeds much faster than that in nonporous $\mathrm{SiC}$ due to weak pore structure modification.

\section{Conclusion}

Modelling based on experimental data on the diffusion of erbium and vanadium in porous silicon carbide at 1700 and $2200^{\circ} \mathrm{C}$ shows that this process is greatly affected by the diffusion of vacancies, which is a typical process in the material with vacancy-related porosity under thermal treatment. Consideration of the vacancy redistribution under annealing allows for satisfactory describing the dopant diffusion in porous $\mathrm{SiC}$ and yields basic diffusion parameters. The vacancy diffusion coefficient on the surface of the pore walls appears to exceed that in the bulk of the material by an order of magnitude. The values of "bulk" diffusion coefficients obtained in this work agree with the data obtained for typical dopants in $\mathrm{SiC}$ (see, e.g., [25]). Generally, it appears that the consideration of vacancy redistribution during hightemperature annealing allows for correct description of diffusion in a porous semiconductor.

\section{Appendix}

Equations for the functions $C_{i j}(x, y, z, t)$ and $V_{i}(x, y, z, t)$ $(i \geq 0, j \geq 0)$ have been solved by the standard Fourier approach (see, e.g., the work by Carslaw and Jaeger [26]).
Solutions of (7) with conditions (8) can be written in the form:

$$
\begin{aligned}
C_{00}( & x, y, z, t) \\
= & -\frac{2 \pi D_{0 C}}{L_{x}^{2} L_{y} L_{z}} \sum_{n=1}^{\infty} n F_{n C} c_{n}(x) c_{n}(y) c_{n}(z) e_{n C}(t) \\
& \times \int_{0}^{t} e_{n C}(-\tau) \int_{0}^{L_{x}} s_{n}(u) \int_{0}^{L_{y}} c_{n}(v) \\
& \times \int_{0}^{L_{z}} c_{n}(w) \frac{D_{C S}(u, v, w, T)}{\bar{V} k T} \frac{\partial \mu(u, v, w, \tau)}{\partial u} d w d v d u d \tau \\
& -\frac{2 \pi D_{0 C}}{L_{x} L_{y}^{2} L_{z}} \sum_{n=1}^{\infty} F_{n C} c_{n}(x) c_{n}(y) c_{n}(z) \\
& \times \int_{0}^{t} e_{n C}(-\tau) \int_{0}^{L_{x}} c_{n}(u) \int_{0}^{L_{y}} s_{n}(v) \\
& \times \int_{0}^{L_{z}} c_{n}(w) \frac{D_{C S}(u, v, w, T)}{\bar{V} k T} \frac{\partial \mu(u, v, w, \tau)}{\partial v} d w d v d u d \tau \\
& \times n e_{n C}(t)-\frac{2 \pi D_{0 C}}{L_{x} L_{y} L_{z}^{2}} \sum_{n=1}^{\infty} n F_{n C} c_{n}(x) \\
& \times c_{n}(y) c_{n}(z) e_{n C}(t) \int_{0}^{t} e_{n C}(-\tau) \int_{0}^{L_{x}} c_{n}(u) \int_{0}^{L_{y}} c_{n}(v) \\
& \times \int_{0}^{L_{z}} s_{n}(w) \frac{D_{C S}(u, v, w, T)}{\bar{V} k T} \frac{\partial \mu(u, v, w, \tau)}{\partial w} d w d v d u d \tau,
\end{aligned}
$$

where $e_{n C}(t)=\exp \left[-\pi^{2} n^{2} D_{0 L} t\left(L_{x}^{-2}+L_{y}^{-2}+L_{z}^{-2}\right)\right], F_{n C}=$ $\int_{0}^{L_{x}} c_{n}(u) \int_{0}^{L_{y}} c_{n}(v) \int_{0}^{L_{z}} f_{C}(u, v, w) c_{n}(w) d w d v d u, c_{n}(x)=\cos$ $(\pi n x / L)$,

$$
\begin{aligned}
& C_{i 0}(x, y, z, t) \\
& =-\frac{2 \pi D_{0 C}}{L_{x}^{2} L_{y} L_{z}} \sum_{n=1}^{\infty} n F_{n C} c_{n}(x) c_{n}(y) c_{n}(z) e_{n C}(t) \\
& \quad \times \int_{0}^{t} e_{n C}(-\tau) \int_{0}^{L_{x}} s_{n}(u) \int_{0}^{L_{y}} c_{n}(v) \\
& \quad \times \int_{0}^{L_{z}} c_{n}(w) g_{L}(u, v, w, T)
\end{aligned}
$$




$$
\begin{aligned}
& \times \frac{\partial C_{i-10}(u, v, w, \tau)}{\partial u} d w d v d u d \tau \\
& -\frac{2 \pi D_{0 C}}{L_{x} L_{y}^{2} L_{z}} \sum_{n=1}^{\infty} F_{n C} c_{n}(x) c_{n}(y) \\
& \times c_{n}(z) \int_{0}^{t} e_{n C}(-\tau) \int_{0}^{L_{x}} c_{n}(u) \int_{0}^{L_{y}} s_{n}(v) \\
& \times \int_{0}^{L_{z}} c_{n}(w) g_{L}(u, v, w, T) \\
& \times \frac{\partial C_{i-10}(u, v, w, \tau)}{\partial v} d w d v d u d \tau \\
& \times n e_{n C}(t)-\frac{2 \pi D_{0 C}}{L_{x} L_{y} L_{z}^{2}} \sum_{n=1}^{\infty} n F_{n C} c_{n}(x) c_{n}(y) c_{n}(z) \\
& \times e_{n C}(t) \int_{0}^{t} e_{n C}(-\tau) \int_{0}^{L_{x}} c_{n}(u) \int_{0}^{L_{y}} c_{n}(v) \int_{0}^{L_{z}} s_{n}(w) \\
& \times g_{L}(u, v, w, T) \frac{\partial C_{i-10}(u, v, w, \tau)}{\partial w} d w d v d u d \tau,
\end{aligned}
$$

where $i \geq 1, s_{n}(x)=\sin (\pi n x / L)$,

$$
\begin{aligned}
& C_{01}(x, y, z, t) \\
& =-\frac{2 \pi D_{0 C}}{L_{x}^{2} L_{y} L_{z}} \sum_{n=1}^{\infty} n F_{n C} c_{n}(x) c_{n}(y) c_{n}(z) e_{n C}(t) \\
& \times \int_{0}^{t} e_{n C}(-\tau) \int_{0}^{L_{x}} s_{n}(u) \int_{0}^{L_{y}} c_{n}(v) \int_{0}^{L_{z}} c_{n}(w) \\
& \times \frac{C_{00}^{\gamma}(u, v, w, \tau)}{P^{\gamma}(u, v, w, T)} \frac{\partial C_{00}(u, v, w, \tau)}{\partial v} d w d v d u d \tau \\
& -\frac{2 \pi D_{0 C}}{L_{x} L_{y}^{2} L_{z}} \sum_{n=1}^{\infty} F_{n C} c_{n}(x) c_{n}(y) \\
& \times n \int_{0}^{t} e_{n C}(-\tau) \int_{0}^{L_{x}} c_{n}(u) \int_{0}^{L_{y}} s_{n}(u) \int_{0}^{L_{z}} c_{n}(w) \\
& \times \frac{C_{00}^{\gamma}(u, v, w, \tau)}{P^{\gamma}(u, v, w, T)} \frac{\partial C_{00}(u, v, w, \tau)}{\partial v} d w d v d u d \tau \\
& \times c_{n}(z) e_{n C}(t)-\frac{2 \pi D_{0 C}}{L_{x} L_{y} L_{z}^{2}} \sum_{n=1}^{\infty} n c_{n}(x) c_{n}(y) c_{n}(z) e_{n C}(t) \\
& \times \int_{0}^{t} e_{n C}(-\tau) \int_{0}^{L_{x}} c_{n}(u) \int_{0}^{L_{y}} c_{n}(u) \int_{0}^{L_{z}} s_{n}(w) \\
& \times F_{n C} \frac{C_{00}^{\gamma}(u, v, w, \tau)}{P^{\gamma}(u, v, w, T)} \frac{\partial C_{00}(u, v, w, \tau)}{\partial v} d w d v d u d \tau,
\end{aligned}
$$

$C_{02}(x, y, z, t)$

$$
=-\frac{2 \pi D_{0 C}}{L_{x}^{2} L_{y} L_{z}} \sum_{n=1}^{\infty} n F_{n C} c_{n}(x) c_{n}(y) c_{n}(z) e_{n C}(t)
$$

$$
\begin{aligned}
& \times \int_{0}^{t} e_{n C}(-\tau) \int_{0}^{L_{x}} s_{n}(u) \int_{0}^{L_{y}} c_{n}(v) \\
& \times \int_{0}^{L_{z}} \frac{C_{00}^{\gamma-1}(u, v, w, \tau)}{P^{\gamma}(u, v, w, T)} \\
& \times\left[C_{01}(u, v, w, \tau) \frac{\partial C_{00}(u, v, w, \tau)}{\partial u}\right. \\
& \left.\quad+C_{00}(u, v, w, \tau) \frac{\partial C_{01}(u, v, w, \tau)}{\partial u}\right] \\
& \times c_{n}(w) d w d v d u d \tau-\frac{2 \pi D_{0 C}}{L_{x} L_{y}^{2} L_{z}} \sum_{n=1}^{\infty} n F_{n C} c_{n}(x) \\
& \times c_{n}(y) c_{n}(z) e_{n C}(t) \int_{0}^{t} e_{n C}(-\tau) \int_{0}^{L_{x}} c_{n}(u) \\
& \times \int_{0}^{L_{y}} s_{n}(v) \int_{0}^{L_{z}} c_{n}(w) \frac{C_{00}^{\gamma-1}(u, v, w, \tau)}{P^{\gamma}(u, v, w, T)} \\
& \times\left[C_{01}(u, v, w, \tau) \frac{\partial C_{00}(u, v, w, \tau)}{\partial v}\right. \\
& \left.+C_{00}(u, v, w, \tau) \frac{\partial C_{01}(u, v, w, \tau)}{\partial v}\right] d w d v d u d \tau
\end{aligned}
$$$$
-\frac{2 \pi D_{0 C}}{L_{x} L_{y} L_{z}^{2}} \sum_{n=1}^{\infty} n F_{n C} c_{n}(x) c_{n}(y)
$$

$$
\begin{aligned}
& \times c_{n}(z) e_{n C}(t) \int_{0}^{t} e_{n C}(-\tau) \int_{0}^{L_{x}} c_{n}(u) \\
& \times \int_{0}^{L_{y}} \int_{0}^{L_{z}} \frac{C_{00}^{\gamma-1}(u, v, w, \tau)}{P^{\gamma}(u, v, w, T)} \\
& \times\left[C_{01}(u, v, w, \tau) \frac{\partial C_{00}(u, v, w, \tau)}{\partial w}\right. \\
& \left.\quad+C_{00}(u, v, w, \tau) \frac{\partial C_{01}(u, v, w, \tau)}{\partial w}\right] \\
& \times s_{n}(w) d w c_{n}(v) d v d u d \tau
\end{aligned}
$$

$$
\begin{aligned}
& C_{11}(x, y, z, t) \\
& =-\frac{2 \pi D_{0 C}}{L_{x}^{2} L_{y} L_{z}} \sum_{n=1}^{\infty} n F_{n C} c_{n}(x) c_{n}(y) c_{n}(z) e_{n C}(t) \\
& \times \int_{0}^{t} e_{n C}(-\tau) \int_{0}^{L_{x}} s_{n}(u) \int_{0}^{L_{y}} c_{n}(v) \\
& \times \int_{0}^{L_{z}}\left[g_{L}(u, v, w, T) \frac{\partial C_{01}(u, v, w, \tau)}{\partial u}\right. \\
& +\frac{C_{00}^{\gamma}(u, v, w, \tau)}{P^{\gamma}(u, v, w, T)} \frac{\partial C_{10}(u, v, w, \tau)}{\partial u} \\
& +C_{10}(u, v, w, \tau) \frac{C_{00}^{\gamma-1}(u, v, w, \tau)}{P^{\gamma}(u, v, w, T)} \\
& \times \frac{\partial C_{00}(u, v, w, \tau)}{\partial u}+g_{L}(u, v, w, T)
\end{aligned}
$$




$$
\begin{aligned}
& \left.\times \frac{C_{00}^{\gamma}(u, v, w, \tau)}{P^{\gamma}(u, v, w, T)} \frac{\partial C_{00}(u, v, w, \tau)}{\partial u}\right] \\
& \times \int_{0}^{L_{z}} c_{n}(w) \frac{D_{V S}(u, v, w, T)}{\bar{V} k T} \\
& \times c_{n}(w) d w d v d u d \tau-\frac{2 \pi D_{0 C}}{L_{x} L_{y}^{2} L_{z}} \sum_{n=1}^{\infty} n F_{n C} c_{n}(x) c_{n} \\
& \times(y) c_{n}(z) e_{n C}(t) \int_{0}^{t} e_{n C}(-\tau) \int_{0}^{L_{x}} c_{n}(u) \\
& \times \int_{0}^{L_{y}} s_{n}(v) \int_{0}^{L_{z}} c_{n}(w) \\
& \times\left[g_{L}(u, v, w, T) \frac{\partial C_{01}(u, v, w, \tau)}{\partial v}\right. \\
& +\frac{C_{00}^{\gamma}(u, v, w, \tau)}{P^{\gamma}(u, v, w, T)} \frac{\partial C_{10}(u, v, w, \tau)}{\partial v} \\
& +C_{10}(u, v, w, \tau) \frac{C_{00}^{\gamma-1}(u, v, w, \tau)}{P^{\gamma}(u, v, w, T)} \\
& \times \frac{\partial C_{00}(u, v, w, \tau)}{\partial v}+g_{L}(u, v, w, T) \\
& \left.\times \frac{\partial C_{00}(u, v, w, \tau)}{\partial v} \frac{C_{00}^{\gamma}(u, v, w, \tau)}{P^{\gamma}(u, v, w, T)}\right] d w d v d u d \tau \\
& -\frac{2 \pi D_{0 C}}{L_{x} L_{y} L_{z}^{2}} \sum_{n=1}^{\infty} n c_{n}(x) c_{n}(y) c_{n}(z) e_{n C}(t) \\
& \times \int_{0}^{t} e_{n C}(-\tau) F_{n C} \int_{0}^{L_{x}} s_{n}(u) \\
& \times \int_{0}^{L_{y}} c_{n}(v) \int_{0}^{L_{z}} c_{n}(w) \\
& \times\left[g_{L}(u, v, w, T)\right. \\
& \times \frac{\partial C_{01}(u, v, w, \tau)}{\partial w}+\frac{\partial C_{10}(u, v, w, \tau)}{\partial w} \\
& \times \frac{C_{00}^{\gamma}(u, v, w, \tau)}{P^{\gamma}(u, v, w, T)}+C_{10}(u, v, w, \tau) \\
& \times \frac{C_{00}^{\gamma-1}(u, v, w, \tau)}{P^{\gamma}(u, v, w, T)} \frac{\partial C_{00}(u, v, w, \tau)}{\partial w} \\
& +\frac{\partial C_{00}(u, v, w, \tau)}{\partial w} g_{L}(u, v, w, T) \\
& \left.\times \frac{C_{00}^{\gamma}(u, v, w, \tau)}{P^{\gamma}}(u, v, w, T)\right] d w d v d u d \tau, \\
& V_{0}(x, y, z, t) \\
& =-\frac{2 \pi D_{0 V}}{L_{x}^{2} L_{y} L_{z}} \sum_{n=1}^{\infty} n F_{n V} c_{n}(x) c_{n}(y) c_{n}(z) \\
& \times e_{n V}(t) \int_{0}^{t} e_{n V}(-\tau) \int_{0}^{L_{x}} s_{n}(u) \int_{0}^{L_{y}} c_{n}(v) \\
& \times \frac{\partial \mu(u, v, w, \tau)}{\partial u} d w d v d u d \tau-\frac{2 \pi D_{0 V}}{L_{x} L_{y}^{2} L_{z}} \\
& \times \sum_{n=1}^{\infty} F_{n V} c_{n}(x) c_{n}(y) c_{n}(z) \int_{0}^{t} e_{n C}(-\tau) \\
& \times \int_{0}^{L_{x}} c_{n}(u) \int_{0}^{L_{y}} s_{n}(v) \int_{0}^{L_{z}} c_{n}(w) \\
& \times \frac{D_{V S}(u, v, w, T)}{\bar{V} k T} \frac{\partial \mu(u, v, w, \tau)}{\partial v} d w d v d u d \tau \\
& \times n e_{n V}(t)-\frac{2 \pi D_{0 V}}{L_{x} L_{y} L_{z}^{2}} \\
& \times \sum_{n=1}^{\infty} n F_{n V} c_{n}(x) c_{n}(y) c_{n}(z) e_{n V}(t) \\
& \times \int_{0}^{t} e_{n V}(-\tau) \int_{0}^{L_{x}} c_{n}(u) \int_{0}^{L_{y}} c_{n}(v) \\
& \times \int_{0}^{L_{z}} s_{n}(w) \frac{D_{V S}(u, v, w, T)}{\bar{V} k T} \\
& \times \frac{\partial \mu(u, v, w, \tau)}{\partial w} d w d v d u d \tau \\
& V_{i}(x, y, z, t) \\
& =-\frac{2 \pi D_{0 V}}{L_{x}^{2} L_{y} L_{z}} \sum_{n=1}^{\infty} n F_{n V} c_{n}(x) c_{n}(y) c_{n}(z) \\
& \times e_{n V}(t) \int_{0}^{t} e_{n V}(-\tau) \int_{0}^{L_{x}} s_{n}(u) \int_{0}^{L_{y}} c_{n}(v) \\
& \times \int_{0}^{L_{z}} c_{n}(w) g_{V}(u, v, w, T) \frac{\partial V_{i-1}(u, v, w, \tau)}{\partial u} d w d v d u d \tau \\
& -\frac{2 \pi D_{0 V}}{L_{x} L_{y}^{2} L_{z}} \sum_{n=1}^{\infty} F_{n V} c_{n}(x) \\
& \times c_{n}(y) c_{n}(z) \int_{0}^{t} e_{n V}(-\tau) \int_{0}^{L_{x}} c_{n}(u) \\
& \times \int_{0}^{L_{y}} s_{n}(v) \int_{0}^{L_{z}} c_{n}(w) g_{V}(u, v, w, T) \\
& \times \frac{\partial V_{i-1}(u, v, w, \tau)}{\partial v} d w d v d u d \tau \\
& \times n e_{n V}(t) \\
& -\frac{2 \pi D_{0 V}}{L_{x} L_{y} L_{z}^{2}} \sum_{n=1}^{\infty} n F_{n V} c_{n}(x) c_{n}(y) c_{n}(z) \\
& \times e_{n V}(t) \int_{0}^{t} e_{n V}(-\tau) \int_{0}^{L_{x}} c_{n}(u)
\end{aligned}
$$




$$
\begin{aligned}
& \times \int_{0}^{L_{y}} c_{n}(v) \int_{0}^{L_{z}} s_{n}(w) g_{V}(u, v, w, T) \\
& \times \frac{\partial V_{i-1}(u, v, w, \tau)}{\partial w} d w d v d u d \tau .
\end{aligned}
$$

\section{Acknowledgments}

This work was partly supported by the grant of the President of Russia (Project no. MK-548.2010.2).

\section{References}

[1] A. V. Bolotnikov, P. G. Muzykov, and T. S. Sudarshan, "Investigation of two-branch boron diffusion fromvapor phase in $n$ type 4H-SiC," Applied Physics Letters, vol. 93, no. 5, Article ID 052101, 3 pages, 2008.

[2] A. V. Bolotnikov, P. G. Muzykov, A. E. Grekov, and T. S. Sudarshan, "Improvement of $4 \mathrm{H}-\mathrm{SiC}$ power $\mathrm{p}-\mathrm{i}-\mathrm{n}$ diode switching performance through local lifetime control using boron diffusion," IEEE Transactions on Electron Devices, vol. 54, no. 6, pp. 1540-1544, 2007.

[3] E. Friedland, J. B. Malherbe, N. G. van der Berg et al., "Study of silver diffusion in silicon carbide," Journal of Nuclear Materials, vol. 389, no. 2, pp. 326-331, 2009.

[4] A. Y. Vodakov and E. N. Mokhov, "Diffusion and solubility of impurities in silicon carbide," in Proceedings of the 3rd International Conference on Silicon Carbide 1973, R. C. Marshall, J. W. Faust Jr., and C. E. Ryan, Eds., pp. 508-520, South Carolina University Press, Florida, Fla, USA, 1974.

[5] A. Y. Vodakov and E. N. Mokhov, "Point defects in silicon carbide," Institute of Physics Conference Series, vol. 137, pp. 197-206, 1994.

[6] Y. Gao, S. I. Soloviev, and T. S. Sudarshan, "Planar 4H- and $6 \mathrm{H}-\mathrm{SiC}$ p-n diodes fabricated by selective diffusion of boron," Solid-State Electronics, vol. 45, no. 12, pp. 1987-1990, 2001.

[7] M. G. Mynbaeva, K. D. Mynbaev, V. A. Ivantsov, A. A. Lavrent'ev, B. A. Grayson, and J. T. Wolan, "Semi-insulating porous SiC substrates," Semiconductor Science and Technology, vol. 18, no. 6, pp. 602-606, 2003.

[8] R. Rurali, E. Hernández, P. Godignon, J. Rebollo, and P. Ordejón, "First-principles studies of the diffusion of B impurities and vacancies in SiC," Physical Review B, vol. 69, no. 12, Article ID 125203, 2004.

[9] R. M. Feenstra and C. E. C. Wood, Eds., Porous Silicon Carbide and Gallium Nitride: Epitaxy, Catalysis, and Biotechnology Applications, John Wiley \& Sons, Chichester, UK, 2008.

[10] M. G. Mynbaeva and K. D. Mynbaev, "Technological Applications of Porous SiC," in Nanocrystals and Quantum Dots of Group IV Semiconductors, T. V. Torchinska and V. Yu. Vorobiev, Eds., pp. 255-276, American Scientific, New York, NY, USA, 2010.

[11] M. G. Mynbaeva, D. A. Bauman, and K. D. Mynbaev, "On the role of vacancies in pore formation in the course of anodizing of silicon carbide," Physics of the Solid State, vol. 47, no. 9, pp. 1630-1636, 2005.

[12] J. Bai, G. Dhanaraj, P. Gouma, M. Dudley, and M. Mynbaeva, "Porous SiC for HT chemical sensing devices: an assessment of its thermal stability," Materials Science Forum, vol. 457-460, no. 2, pp. 1479-1482, 2004.

[13] M. Mynbaeva, A. Lavrent'ev, I. Kotousova, A. Volkova, K. Mynbaev, and A. Lebedev, "On current limitations in porous
SiC applications," Materials Science Forum, vol. 483-485, pp. 269-272, 2005.

[14] V. Glukhanyuk and A. Kozanecki, "Site selective studies of $\mathrm{Er}^{3+}$ emission centers in Er-implanted 6H-SiC," Applied Physics Letters, vol. 89, no. 21, Article ID 211114, 2006.

[15] N. T. Son, P. Carlsson, A. Gällström, B. Magnusson, and E. Janźn, "Deep levels and carrier compensation in V-doped semi-insulating 4H-SiC," Applied Physics Letters, vol. 91, no. 20, Article ID 202111, 2007.

[16] P. G. Cheremskoy, V. V. Slezov, and V. I. Betekhtin, Pores in Solids, Energoatomizdat, Moscow, Russia, 1990.

[17] M. G. Mynbaeva, E. N. Mokhov, A. A. Lavrent'ev, and K. D. Mynbaev, "High-temperature diffusion doping of porous silicon carbide," Technical Physics Letters, vol. 34, no. 9, pp. 731733, 2008 .

[18] M. Kitayama, T. Narushima, and A. M. Glaeser, "Wulff shape of alumina: II, experimental measurements of pore shape evolution rates," Journal of the American Ceramic Society, vol. 83, no. 10, pp. 2572-2583, 2000.

[19] Z. Yu. Gotra, Technology of Microelectronic Devices, Radio and Communications, Moscow, Russia, 1991.

[20] E. I. Zorin, P. V. Pavlov, and D. I. Tetelbaum, Ion Doping of Semiconductors, Energiya, Moscow, Russia, 1975.

[21] H. Ryssel and I. Ruge, Ion Implantation, Teubner, Stuttgart, Germany, 1978.

[22] E. L. Pankratov, "Influence of the spatial, temporal, and concentrational dependence of the diffusion coefficient on dopant dynamics: optimization of annealing time," Physical Review B, vol. 72, no. 7, Article ID 075201, 2005.

[23] E. L. Pankratov and B. Spagnolo, "Optimization of impurity profile for p-n-junction in heterostructures," European Physical Journal B, vol. 46, no. 1, pp. 15-19, 2005.

[24] E. L. Pankratov, "Dopant diffusion dynamics and optimal diffusion time as influenced by diffusion-coefficient nonuniformity," Russian Microelectronics, vol. 36, no. 1, pp. 33-39, 2007.

[25] V. A. Dmitriev and M. G. Spencer, "SiC fabrication technology: growth and doping," Semiconductors and Semimetals, vol. 52, pp. 21-75, 1998.

[26] H. S. Carslaw and J. C. Jaeger, Conduction of Heat in Solids, Oxford University Press, Oxford, UK, 1964. 

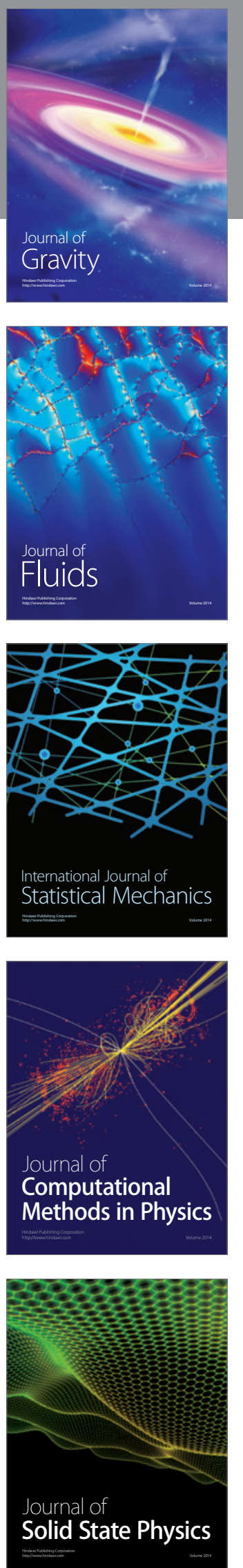

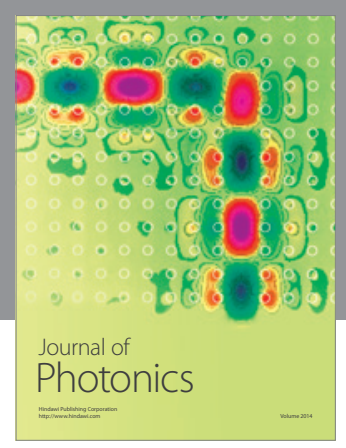

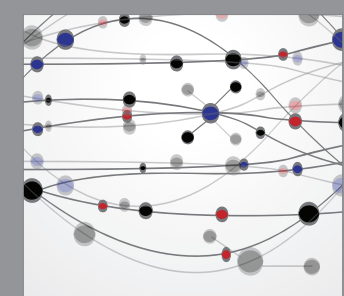

The Scientific World Journal
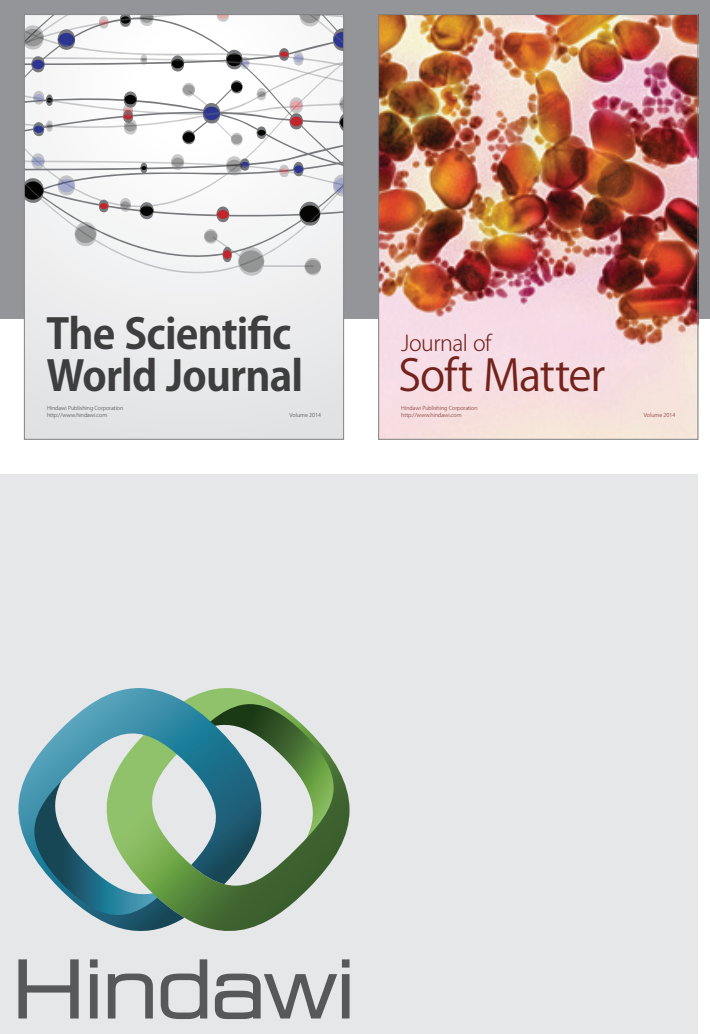

Submit your manuscripts at

http://www.hindawi.com
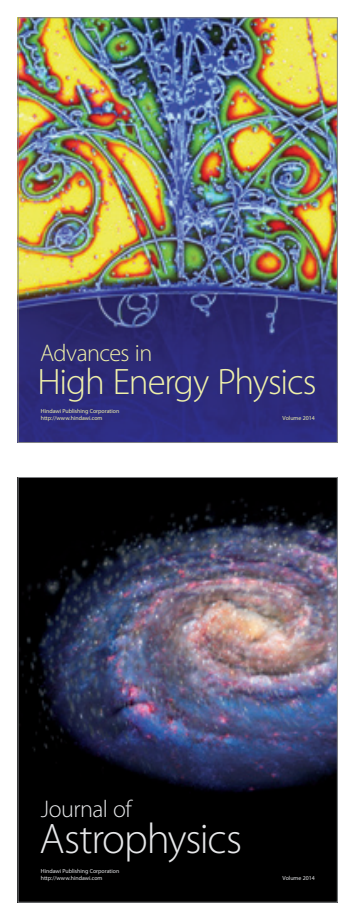
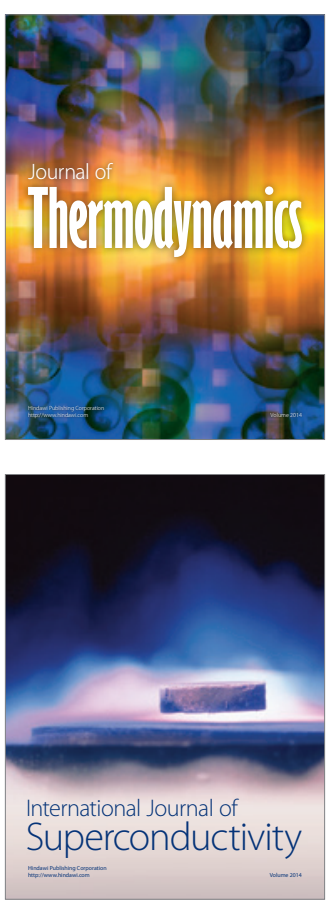
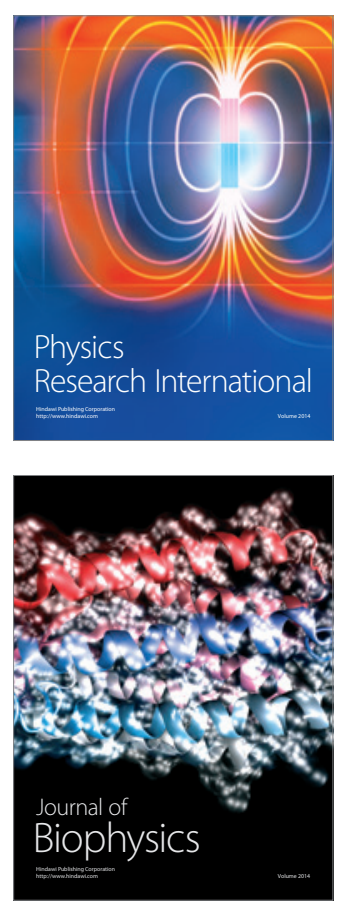
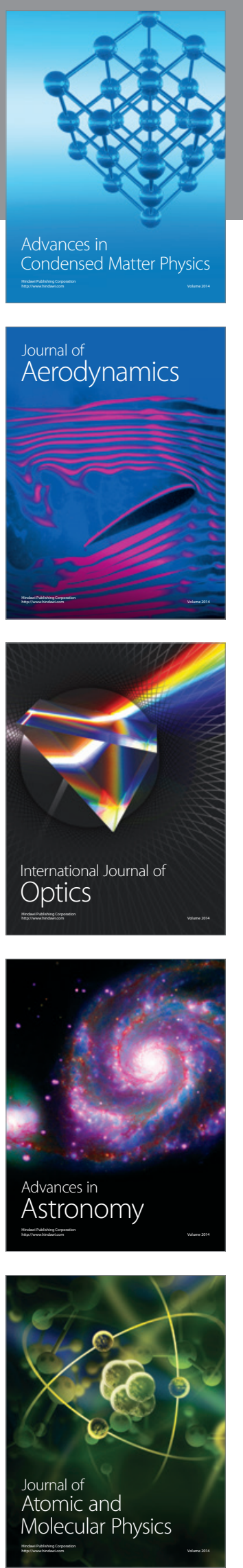